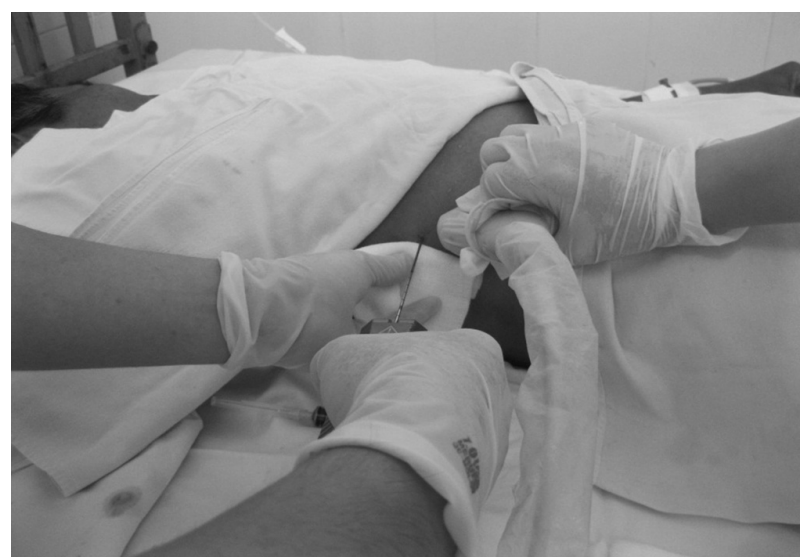

Abstract IDDF2018-ABS-0033 Figure 1

Results Distribution in 93 patients according to the Metavir score was F1: $\mathrm{n}=13(6.6 \mathrm{kPa}) ; \mathrm{F} 2: \mathrm{n}=24 \quad(8.6 \mathrm{kPa}) ; \mathrm{F} 3$ : $\mathrm{n}=31 \quad(18.5 \mathrm{kPa})$ and $\mathrm{F} 4: \mathrm{n}=25(40.8 \mathrm{kPa}) \quad(\mathrm{p}<0.001)$. To diagnose liver fibrosis moderate $(\mathrm{F} \geq \mathrm{F} 2)$ : AUROC: 0.86 . Threshold value is $7.9 \mathrm{kPa}$. Sensitivity (Se), specificity (Sp), (positive predictive value) PPV and negative predictive value (NPV) were $80 \%$; 90\%, 91\%; and $72 \%$ for $\mathrm{F} \geq \mathrm{F} 2$. To diagnose liver fibrosis severity $(\mathrm{F} \geq \mathrm{F} 3)$ : AUROC: 0.91 . Threshold value is $11.3 \mathrm{kPa}$. Se, Sp, PPV and NPV were $86.8 \%$; $81 \%$; $82 \%$; and $85 \%$ for $\mathrm{F} \geq \mathrm{F} 3$.

Conclusions Fibroscan is effective to assess liver fibrosis in patients with ALD. Instant screening of liver fibrosis in heavy drinkers is feasible without liver biopsy.

\section{IDDF2018-ABS-0035 ROLE OF CYTOKINES IN ALCOHOLIC LIVER DISEASE}

Le Thi Thu Hien*, Le Quoc Tuan. Department of Internal Medicine, Thai Nguyen University of Medicine and Pharmacy, Vietnam

\subsection{6/gutjnl-2018-IDDFabstracts. 195}

Background Alcohol liver disease (ALD) is a major cause of morbidity and mortality worldwide. Chronic alcohol consumption leads to hepatocellular injury, fat accumulation, liver inflammation, liver fibrosis and cirrhosis or hepatocellular carcinoma. Cytokines are inflammatory mediators and one of the key factors in the various aspects of the pathophysiology of ALD.

Aims To investigate cytokines in patients with ALD

Methods Our study was conducted in Thai Nguyen Nation Hospital and 103 Military Hospital. Tumour necrosis factor alpha (TNF- $\alpha$ ), transforming growth factor beta (TGF- $\beta$ ), interleukin - $1 \beta$ (IL-1 $\beta$ ) and interleukin-12 (IL-12) were measured in 105 cases of ALD and 40 healthy volunteers which were regarded as the control group. Using ELISA kit supplied by Wkea Med Supplies Corp, China. We used median for comparison because of non-standard distribution.

Results Age of 45-59 accounts for the highest rate of $50.5 \%$. Compared to the control group, patients with ALD showed significantly lower TGF- $\beta(1172.28 \mathrm{ng} / \mathrm{mL}$ vs $110829.44 \mathrm{ng} /$ $\mathrm{mL}, \quad \mathrm{p}<0.001), \quad$ TNF- $\alpha \quad(158.8 \mathrm{pg} / \mathrm{mL} \quad$ vs $173.64 \mathrm{pg} / \mathrm{mL}$, $\mathrm{p}<0.005)$. ALD patients had significantly higher IL-1 $\beta$ (14.56 ng/L mmol/L vs $3.19 \mathrm{ng} / \mathrm{L}, \mathrm{p}<0.001)$, IL-12 (27.47 ng/
$\mathrm{L}$ vs $4.0 \mathrm{ng} / \mathrm{L}, \mathrm{p}<0.001)$ than that in the control group. Levels of serum TGF- $\beta$ and IL-1 $\beta$ are associated with liver fibrosis stage $(\mathrm{p}<0.001)$. Serum IL-12 and TNF- $\alpha$ levels reflected the different stages of alcoholic liver disease $(\mathrm{p}<0.05)$.

Conclusions Cytokines play important roles in the development of ALD. They have the potential to be biomarkers of alcoholic liver disease. More studies are needed to increase the understanding of the pathogenesis of ALD to open new therapeutic avenues for ALD.

\section{IDDF2018-ABS-0042 OVERT HEPATIC ENCEPHALOPATHY IN EXTRA HEPATIC PORTAL VENOUS OBSTRUCTION WITH LUPUS NEPHRITIS IN YOUNG FEMALE}

Apurva Shah*. Apollo Hospitals International Limited, Ahmedabad, India

\subsection{6/gutjnl-2018-IDDFabstracts. 196}

Background Spontaneous hepatic encephalopathy is very uncommon in extrahepatic portal venous obstruction (EHPVO), except when large spontaneous portosystemic shunts have developed. We present a case of a young female who was diagnosed as EHPVO and now presented as overt hepatic encephalopathy due to spontaneous lienorenal shunt and later she was diagnosed with lupus nephritis.

Methods A 36-year-old female hospitalised in July 2007 with complaints of disorientation and personality changes which were present for last one month. She was diagnosed EHPVO in 1998 when she had left hypochondriac pain, bilateral oedema feet and oedema over the face with a history of infertility and coil embolization for splenic artery aneurysm. On examination, she was conscious, not oriented and focal neurological signs were absent. General and abdominal examination revealed pallor, bilateral pitting oedema feet and splenomegaly. Her investigations revealed anaemia, hypothyroidism, and albumin/globulin ratio reversal. CT abdomen had shown shrunken liver, portal vein thrombosis, coils in splenic artery with splenomegaly and extensive portosystemic collaterals. Gastroscopy revealed antral gastritis, no varices. Liver biopsy revealed portal fibrosis, no cirrhosis.

Results Over 48 hours she responded to anti encephalopathic measures. She was apparently well for three years with thyroid hormone replacement, diuretics and lactulose. She had been hospitalised again in 2010 with anasarca and massive right-sided pleural effusion. She had nephrotic range proteinuria and diagnosed as lupus nephritis by renal biopsy and was started on oral steroids. She had developed second episode of overt encephalopathy which was spontaneous in 2010, and she had responded to medical treatment for encephalopathy.

Conclusions Though she was diagnosed with lupus nephritis many years after the diagnosis of EHPVO, portal vein thrombosis may be due to acquired thrombophilic disorder - antiphospholipid syndrome which was not recognised earlier. Presence of weakly positive lupus antibody, history of infertility, portal vein thrombosis and nephrotic range proteinuria may represent a spectrum of manifestation of antiphospholipid syndrome in this case. Defective acquired fibrinolysis and antiphospholipid syndrome are proposed as a cause of portal vein thrombosis in SLE. Surgical ligation of portosystemic shunt is occasionally of benefit when hepatic encephalopathy is refractory to other treatment. 